\title{
El Parto Prematuro en el I.S.S. Valle (Informe Preliminar)
}

\author{
Dra. Sonia Pazmiño de Osorio, MD.
}

\section{AGRADECIMIENTOS}

A todo el personal médico, paramédico y administrativo que ha intervenido en el estudio. A los internos y estudiantes de Medicina de la Universidad Libre; a la Doctora Blanca Beatrlz Garcla, Jefe de Laboratorio del U.P.I.R.U.U., al doctor Jorge Paredes, Jefe de la División de Salud Ocupacional de la Seccional; al Doctor Alfredo Buitrago, Jefe de la Sección de Medicina del Trabajo; al Doctor Adolfo Congote, Jefe del Departamento Materno Infantil del U.P.I.R.U.U.

Es con su participación y apoyo decidido como se está logrando efectuar este trabajo.

\section{INTRODUCCION}

Estudios realizados en Latinoamérica han comprobado la importancia que tiene el parto prematuro como causa de muerte en el neonato. En la investigación Latinoamericana en la Niñez (Puffer, 1971), se encontró que de la mitad a las dos terceras partes de las muertes neonatales ocurrieron en prematuros. En Cali correspondió a la mitad de las muertes.

Desafortunadamente se desconoce la dimensión del problema a nivel nacional; sin embargo, se puede considerar como importante, dada la similitud de las condiciones sociales y económicas de los países latinoamericanos y a haberse detectado como patología severa en la población del I.S.S. del Valle.

\section{EI Problema en el I.S.S. Valle}

En la Seccional del Valle del Cauca, con registros establecidos desde 1981, a través del Programa de Atención Materna se ha logrado detectar que el parto prematuro es la primera causa de Mortalidad Neonatal y la amenaza de parto prematuro es la mayor causa de la hospitalización materna.

INCIDENCIA DE PARTO PREMATURO

I.S.S. VALLE

\begin{tabular}{|lll|}
\hline Clínica & $\begin{array}{c}1982 \\
(\%)\end{array}$ & $\begin{array}{c}1983^{*} \\
(\%)\end{array}$ \\
\hline R.U.U. - Cali & 5.9 & 8.5 \\
Palmira & 4.4 & 5.5 \\
Buga & 1.6 & 0.4 \\
Tuluá & 1.7 & 1.1 \\
\hline Total & 5.1 & 7.1 \\
\hline
\end{tabular}

Primer Semestre 
Ha sido notable el aumento en el primer semestre de este año.

Debido a la alta morbilidad y mortalidad del prematuro, que requiere equipo y personal especializado que no están disponibles en nuestra clínica, y a la posibilidad de evitar la mayoría de los partos prematuros con un buen manejo terapéutico, se ha diseñado una campaña de detección y tratamiento oportuno de la entidad.

\section{PLAN PROPUESTO}

La campaña incluye tres aspectos:

\section{Aspecto Preventivo:}

Establecer la divulgación e información de la entidad, tanto a las maternas en el control prenatal como al personal médico y paramédico de los diferentes niveles de atención de la institución.

\section{Aspecto Curativo:}

Normatización del tratamiento con la adaptación y adopción de las pautas de manejo, promulgadas por la Organización Mundial de la Salud. Se incluye el manejo ambulatorio e intrahospitalario, así como la solicitud de los recursos necesarios a la administración del Instituto.

\section{Aspecto Investigativo:}

Aspecto fundamental que permitirá retroalimentar los dos primeros.

Los aspectos 1 y 2 se están llevando a cabo de forma irregular, básicamente por dificultades de índole administrativo. La investigación, si bien ha tenido tropiezos, se desarrolla sin mayores contratiempos y terminará a mediados de 1984.

Aspecto Investigativo: Estudio de Factores asociados con el Parto Prematuro

Una de las motivaciones que más ha influido para llevarlo a cabo, a pesar de que aparentemente sobre esta entidad ya todo se sabe, es haber encontrado que es significativamente más frecuente entre nuestras maternas trabajadoras (afiliadas) comparada con las beneficiarias.

Es muy importante destacar la colaboración prestada por los Doctores Federico Rivero y Rodrigo Muñoz en esta parte del trabajo.

\section{OBJETIVOS}

1. Establecer la frecuencia de algunas características personales y ocupacionales de las maternas que presenten la entidad.

2. De acuerdo al conocimiento del Objetivo 1, determinar posibles acciones preventivas primarias en el control prenatal.

\section{METODOLOGIA}

De la población gestante que asista a la Clínica Rafael Uribe Uribe del I.S.S. de Cali, durante 6 meses se toma dos grupos de pacientes: las que presentan amenaza de o parto prematuro, que se denominan casos y una muestra aleatoria de gestantes con parto a término, que se denominan controles.

- De los casos:

A toda paciente con la entidad a estudiar se le aplicará un formulario que investiga las características personales, tipo de ocupación, vivienda, enfermedad previa, antecedentes ginecobstétricos y patología durante el embarazo actual.

Se le toman algunos exámenes de laboratorio como son: $\mathrm{Hb}$, serología, parcial de orina, urocultivo, proteínás y glicemia. Número calculado de 475. 
- De los controles:

Se toma al azar pacientes apareadas por calidad del usuario, afiliada o beneficiaria y por grupos de edad.

Se les aplica igual formulario y exámenes de laboratorio que a los casos. Estas pacientes no deben haber presentado durante este embarazo amenaza de parto prematuro.

Número calculado 950.2 controles por caso.

\section{Desarrollo}

De los casos salen dos grupos: pacientes con causa médica conocida como determinante de la entidad (Grupo D) $y$ las que no presenten causa médica conocida (Grupo C).

De las afiliadas del grupo $C$ se toma una muestra representativa (40) y se les practica visita a su puesto de trabajo.

De las afiliadas del grupo $E$ (controles) se toma una muestra similar apareada por edad $y$ ocupación $y$ se practica visita al puesto de trabajo.

\section{La visita al Puesto}

Ha sido efectuada por el Doctor $\mathrm{Al}$ fredo Buitrago, Jefe de la Sección de Medicina del Trabajo de la División de Salud Ocupacional. El instrumento empleado es el formulario de rutina que se utiliza en la división para estas visitas.

\section{RESULTADOS}

Se presentan a continuación algunos de los resultados preliminares obtenidos del análisis del $20{ }^{\circ}$ o de las pacientes del estudio. Esta evaluación nos permite tener una primera aproximación acerca de la situación de la entidad que se está estudiando y efectuar algunos ajustes a la investigación.

\section{OTRA PATOLOGIA ADEMAS \\ DE LA ENTIDAD}

\begin{tabular}{|lrr|}
\hline Otra Patología & Número & $\%$ \\
\hline Ninguna & 88 & 70.1 \\
Infeccion urinaria & 22 & 16.5 \\
Pre-eclampsia & 5 & 3.9 \\
Ruptura prematura de membranas & 4 & 3.1 \\
Gastritis & 2 & 1.6 \\
Prediabetes-diabetes & 2 & 1.6 \\
Otros (insuficiencia placentaria, & & \\
miomapediculado, intoxicación, & & \\
gemelar). & 4 & 3.2 \\
\hline Total & 127 & 100.0 \\
\hline
\end{tabular}

En la gran mayoría de los casos no se encuentra otra patología detectable, diferente a la amenaza o parto prematuro; cuando ésta se hace aparente es la infección urinaria. Menos frecuente se asocia la toxemia y la ruptura prematura de membranas.

\section{DISTRIBUCION POR PARIDAD}

\begin{tabular}{|ccccc|}
\hline \multirow{2}{*}{ Número de } & \multicolumn{2}{c}{ CASOS } & \multicolumn{2}{c|}{ CONTROLES } \\
\cline { 2 - 5 } & Número & $\%$ & Número & $\%$ \\
\hline 0 & 49 & $39.5^{*}$ & 46 & 19.3 \\
$1-2$ & 58 & 46.8 & 160 & 67.2 \\
3 y más & 17 & 13.7 & 32 & 13.5 \\
\hline Total & 124 & 100.0 & 238 & 100.0 \\
\hline
\end{tabular}

R.R.: 2.7

Chi cuadrado: $16.14 \quad \mathrm{p}<.001$

La gestante que no ha tenido partos tiene tres veces más riesgo de tener una amenaza de parto prematuro.

La gestante que tiene antecedente de aborto previo tiene el doble de riesgo de hacer una amenaza de parto prematuro; si además presenta otra patología, el riesgo aumenta tres veces. 
DISTRIBUCION POR ANTECEDENTES DE ABORTOS

\begin{tabular}{|lcrcr|}
\hline \multirow{2}{*}{ Abortos } & \multicolumn{2}{c}{ CASOS } & \multicolumn{2}{c|}{ CONTROLES } \\
\cline { 2 - 5 } & Número & $\%$ & Número & $\%$ \\
\hline 1 y más & 29 & 23.0 & 29 & 12.0 \\
0 & 97 & 77.0 & 212 & 88.0 \\
\hline Total & 120 & 100.0 & 241 & 100.0 \\
\hline
\end{tabular}

R. R. 2.2

Chi cuadrado: $6.7 \mathrm{p}<.01$

ANTECEDENTE DE AMENAZA DE PARTO PREMATURO

\begin{tabular}{|ccccc|}
\hline \multirow{2}{*}{$\begin{array}{l}\text { Embarazo } \\
\text { A.P.P. }\end{array}$} & \multicolumn{2}{c}{ CASOS } & \multicolumn{2}{c|}{ CONTROLES } \\
\cline { 2 - 5 } & Número & \multicolumn{1}{c}{$\%$} & Número & $\%$ \\
\hline 2 & 3 & 3.0 & 2 & .9 \\
1 & 14 & 13.7 & 6 & 2.7 \\
0 & 85 & 83.3 & 214 & 96.4 \\
\hline Total & 102 & 100.0 & 222 & 100.00 \\
\hline
\end{tabular}

Riesgo relativo sumario: 5.6

Chi cuadrado: $18.6 \% \quad \mathrm{p}<.001$

La gestante que ha presentado una amenaza de parto prematuro tiene $\mathbf{5 . 6}$ veces más riesgo de repetirlo.

VISITA AL PUESTO DE SALUD

\begin{tabular}{|llcc|}
\hline & Casos & Controles \\
\hline \multicolumn{1}{|c}{ Establecidos } & 4 & 12 \\
$\begin{array}{l}\text { Intermedio } \\
\end{array}$ & & \\
& No establecido & 13 & 3 \\
\hline
\end{tabular}

en correción de Yates: 8.03

$$
p<0.01
$$

De los diferentes parámetros observados en la visita al Puesto de Trabajo, como son, la jornada laboral, el tipo de oficio, ambiente, etc., se han encontrado que las gestantes con amenaza o parto prematuro tienen un ritmo de trabajo intenso y permanente durante la jornada de trabajo, sin descansos establecidos en la mañana y en la tarde.

\section{Recomendaciones}

Es necesario analizar permanentemente las causas de enfermedad y muerte de la población materno-infantil de cada una de las instituciones de salud del país, con el objeto de investigar los factores determinantes de las mismas que pudieran disminuirse con medidas preventivas durante el control prenatal.

\section{RESUMEN Y CONCLUSIONES}

Se ha encontrado en el I.S.S., Valle del Cauca, el parto prematuro como determinante de la primera causa de mortalidad neonatal y la amenaza de parto prematuro como la primera causa de morbilidad materna hospitalaria. Se ha diseñado una campaña de detección y detención precoz, con base en las normas divulgadas por la Organización Mundial de la Salud, a todos los niveles de la atención de la gestante tanto en la consulta externa como hospitalaria.

Se presentan resultados preliminares de la investigación que conforma 'ıno de los aspectos de la campaña:

1. El ser primigestante, o tener un antecedente de amenaza de aborto o una amenaza de parto prematuro en embarazo anterior, aumenta considerablemente el riesgo de hacer una amenaza de o un parto prematuro.

2. El ritmo de trabajo de las gestantes, sin descansos intermedios en su jornada laboral, es uno de los parámetros que puede tener importancia definitiva en la frecuencia del parto prematu. ro en las afiliadas. 


\section{PREMATURE DELIVERY AT THE I.S.S.}

\section{SUMMARY AND CONCLUSIONS}

At the I.S.S., Valle del Cauca, the premature delivery has been found to be the first cause of neonatal mortality and the threat of premature delivery is the first cause of maternal hospital morbidity. A detection campaign and a precocious detention have been designed, on basis of the norms divulged by the World Health Organization, at all llevels | of attention for pregnant women in hospitals and external consultation.

The preliminary results are presented in the investigation that conforms one of the aspects of the campaign:

\section{BIBLIOGRAFIA}

1. OPS. Boletín de la Oficina Sanitaria Panamericana. Manejo Perintal de la prematurez. 87: 361, 1979.

2. OPS. Las Condiciones de Salud del Niño en las Américas. Publicación Científica OPS/OMS. No. 381. p. 211, 1979.

3. Clínicas Obstétiricas y Ginecológicas. Vol. 1. 1980. Aspectos Obstétricos del Parto Prematuro. Pág. 17-121.

4. High Risk Prematurity Progestin Treat ment and steroid Studies.

5. Obstetrics \& Gynecology V. 54 No. 4 October 1979. JOHN W.C. JOHNSON MD., PETER A. LEE MD., ANNELIES S. ZACHARY MD. SHIRLEY COLHOUN RN. and CALUDE J. MIGEM MD.

6. The Management of Preterm Labor. TOM P. BARDEN MD. University of Cincinnati College of Medicine reprint.

7. Diddle Aw: Gravid Women at Work. Fetal and maternal mortality, employment Po-
1. To be pregnant for the first time, to have an antecedent of threat of abortion or a threat of premature delivery in a previous pregnancy, increases considerably the risk to have a threat or a premature delivery.

2. The rythm of labor in pregnant women, without intermediate rests, is one of the parameters that have a definite importance in the frequency of premature delivery.

licy, and medico legal aspects. J. Occup. Med. 12: 10, 1970.

8. JOHNSON JWC AUSTIN KL. JONES GS et al: Efficacy of 17 bydroxyprogesterone caprovate in the prevention of premature labor. N. Engl: J. Med. 293675, 1975.

9. MARIRATE M. DEVILLIERS. K.Q. FAIRBROTHER P: Effect of prophylactic out patient administration of fenoterol on the time of onset of spontaneous labor and fetal growth rate in the twin pregnancy Am. J. obst Gynecol 128: 707, 1977.

10. WEEKES, ARL, MEIZIES DN. de BOLR $\mathrm{CH}$; the relative efficacy of bed rest cervical suture and No Treatment in the management of twin Pregnancy. Br. J. Obstet. Gynecol 84: 161, 1977.

11. High Risk Prematurity Progestin Treatment and steroid studies. JOHN WC. JOHNSON, MD, PETER A LEE, ANNELIES S. ZACHARY. Obst. \& Gynecol Vol. 54 No. 4 October, 1979. 